\title{
Probabilistic Congruence for Semistochastic Generative Processes
}

\author{
Ruggero Lanotte and Simone Tini \\ Dipartimento di Scienze della Cultura, Politiche e dell'Informazione, \\ Università dell'Insubria, Via Valleggio 11, I-22100, Como, Italy
}

\begin{abstract}
We propose an SOS transition rule format for the generative model of probabilistic processes. Transition rules are partitioned in several strata, giving rise to an ordering relation analogous to those introduced by Ulidowski and Phillips for classic process algebras. Our rule format guarantees that probabilistic bisimulation is a congruence w.r.t. process algebra operations. Moreover, our rule format guarantees that process algebra operations preserve semistochasticity of processes, i.e. the property that the sum of the probability of the moves of any process is either 0 or 1 . Finally, we show that most of operations of the probabilistic process algebras studied in the literature are captured by our format, which, therefore, has practical applications.
\end{abstract}

\section{Introduction}

Probabilistic process algebras have been introduced in the literature (see, among the others, $[2,3,8,9,10,11,13$ ) to develop techniques dealing with both functional and non-functional aspects of system behavior, such as performance and reliability. Probabilistic transition systems (PTSs, for short), which extend classic labeled transition systems by some mechanism to represent the probabilistic choice, have been employed as a basic semantic model of probabilistic processes. In order to abstract away from irrelevant information on the way that processes compute, several notions of behavioral equivalence and preorder have been considered. Probabilistic bisimulation relates two processes iff they have the same probabilistic branching structure. In the process algebras of [2, 3, 8, 9, 10, 11, 13, ), probabilistic bisimulation is a congruence w.r.t. all operations, which is an important property to fit it into an axiomatic framework.

Usually, PTSs are defined by means of a structural operational semantics [14, 15] (SOS, for short) consisting of a set of transition rules of the form $\frac{\text { premises }}{\text { conclusion }}$, which, intuitively, determine how probabilistic moves of processes can be inferred by probabilistic moves of other processes. A set of syntactical constraints on the transition rules is called a transition rule format [16]. In the area of classic (i.e., non-probabilistic) process algebras, rule formats have been widely employed to fix results holding for classes of process algebras. For instance, several rule formats proposed in the literature ensure that a given behavioral equivalence is a congruence (for a survey see [1]). Other rules formats ensure that a given property of security is preserved by process algebra operations [17, 18. 
An interesting issue is to develop rule formats for probabilistic process algebras. To take a step in this direction, we propose a rule format for process algebras respecting the generative model of probabilistic processes [1], which requires that a single probability distribution is ascribed to all moves of any process. Such a generative model differs w.r.t. the reactive model of probabilistic processes, which requires that the kind of action of any process is chosen nondeterministically, and that, for any action and any process, a probability distribution is ascribed to the moves of that process labeled with that action.

Our format admits transition rules of the following form:

$$
\frac{\left\{x_{i} \stackrel{a_{i}, p_{i}}{\longrightarrow} y_{i} \mid i \in I\right\} \cup\left\{x_{j} \stackrel{A_{j}, p_{j}^{\prime}}{\longrightarrow} \mid j \in J\right\} \cup\left\{x_{h} \stackrel{B_{h}}{\longrightarrow} \mid h \in H\right\}}{f(\vec{x}) \stackrel{a, \frac{\prod_{i \in I} p_{i}}{\Pi_{j \in J}\left(1-p_{j}^{\prime}\right)} \cdot w_{\rho}}{\longrightarrow} t}
$$

Hence, our format extends the classic de Simone format [16] with probability (i.e., a probability value $p$ appears in transition labels), premises $x_{j} \stackrel{A_{j}, p_{j}^{\prime}}{\longrightarrow}$ meaning that the argument $j$ of $f$ performs actions in the set $A_{j}$ with total probability $p_{j}^{\prime}$, and premises $x_{h} \stackrel{B_{h}}{\longrightarrow}$ meaning that the argument $h$ of $f$ performs at least one action in the set $B_{h}$. Then, to give a semantics to a given process algebra, we require that the transition rules are partitioned in $n$ strata $\mathcal{R}_{1}, \ldots, \mathcal{R}_{n}$, for some $n \in \mathbb{N}$. The interpretation is that the moves of a given process $t$ can be inferred from rules in $\mathcal{R}_{i}$ only if no move of $t$ can be inferred from rules in $\mathcal{R}_{j}$, for any $j<i$. Hence, the partitioning gives rise to an ordering relation between transition rules analogous to those introduced for classic process algebras in [19.

We prove that process algebra operations captured by our format preserve semistochasticity of processes, i.e. the property that the sum of the probability of the moves of any process is either 0 or 1 . This is a central issue in the theory of probabilistic processes, since semistochasticity is required by most of authors, such as [3,5, 8, which concentrate on so called semistochastic languages [1].

Then, we prove that probabilistic bisimulation is a congruence w.r.t. all operations captured by our format.

To show that our format has practical applications, we prove that it captures most of operations of the probabilistic process algebras proposed in the literature.

Finally, we prove that our format can be enriched by double testing as in GSOS format [7, and by look ahead as in tyft/tyxt format [12. We discuss also the possibility to admit predicates, as in formats path [4] and panth 20.

We discuss the related work [6], where a very preliminary rule format for the reactive model of probabilistic processes is introduced.

\section{Background}

Let us begin with recalling the model of probabilistic transition systems.

For any set $S$, let $\mathcal{M}(S)$ denote the collection of multisets over $S$.

Definition 1. A probabilistic transition system (PTS, for short) is a triple $(\mathcal{S}, A c t, T)$, where $\mathcal{S}$ is a set of states, Act is a set of actions, and $T \in \mathcal{M}(\mathcal{S} \times$ 
Act $\times(0,1] \times \mathcal{S})$ is a multiset of transitions such that, for all states $s \in \mathcal{S}$, $\sum\left\{|p| \exists a \in\right.$ Act, $\left.s^{\prime} \in S:\left(s, a, p, s^{\prime}\right) \in T \mid\right\} \in[0,1]$.

Def. 1 respects the generative (or full) model of probabilistic processes [11, where a single probability distribution is ascribed to all moves of any process. On the contrary, we recall that the reactive model admits that the kind of action is chosen nondeterministically, i.e. the multiset $T$ satisfies the following property: for all states $s \in \mathcal{S}$ and actions $a \in A c t, \sum\left\{|p| \exists s^{\prime} \in \mathcal{S}:\left(s, a, p, s^{\prime}\right) \in T \mid\right\} \in[0,1]$.

Definition 2. A state $s \in \mathcal{S}$ is semistochastic iff $\sum\left\{|p| \exists a \in A c t, s^{\prime} \in S\right.$ : $\left.\left(s, a, p, s^{\prime}\right) \in T\right\} \in\{0,1\}$. If this sum is 1 then $s$ is stochastic. A PTS is semistochastic iff all its states are semistochastic.

As in $3,5,8$, we concentrate on semistochastic PTSs, which are the semantic model of the so called semistochastic languages [11.

We write $s \stackrel{a, p}{\longrightarrow} s^{\prime}$ to denote that $\left(s, a, p, s^{\prime}\right) \in T$, and we call $s$ and $s^{\prime}$ source and target of the transition, respectively. For a set of actions $A \subseteq A c t$, we write $s \stackrel{A, p}{\longrightarrow}$ to denote that $\sum\left\{|q| \exists a \in A, s^{\prime} \in \mathcal{S}: s \stackrel{a, q}{\longrightarrow} s^{\prime}\right\}=p$. If this multiset is empty, then we write $s \stackrel{A, 0}{\longrightarrow}$. Finally, we write $s \stackrel{A}{\longrightarrow}$ to denote that there is at least one transition $\left(s, a, p, s^{\prime}\right)$ in $T$ with $a \in A$, for some $p$ and $s^{\prime}$.

Before defining probabilistic bisimulation, we need some definitions.

For an equivalence relation $\mathcal{R}$ over $\mathcal{S}$, we write $\mathcal{S} / \mathcal{R}$ to denote the set of equivalence classes induced by $\mathcal{R}$.

Definition 3. $\mu: \mathcal{S} \times$ Act $\times 2^{\mathcal{S}} \rightarrow[0,1]$ is the function given by: $\forall s \in \mathcal{S}$, $\forall a \in A c t, \forall S \subseteq \mathcal{S}$

$$
\mu(s, a, S)=\sum\left\{|p| s \stackrel{a, p}{\longrightarrow} s^{\prime} \text { and } s^{\prime} \in S \mid\right\}
$$

Definition 4. An equivalence relation $\mathcal{R} \subseteq \mathcal{S} \times \mathcal{S}$ is a probabilistic bisimulation if $\left(s_{1}, s_{2}\right) \in \mathcal{R}$ implies: $\forall S \in \mathcal{S} / \mathcal{R}, \forall a \in$ Act,

$$
\mu\left(s_{1}, a, S\right)=\mu\left(s_{2}, a, S\right)
$$

The union of all probabilistic bisimulation is, in turn, a probabilistic bisimulation. We denote it by $\approx$, and we write $s_{1} \approx s_{2}$ for $\left(s_{1}, s_{2}\right) \in \approx$.

Let us recall now the notions of signature and term over a signature.

A signature is a set $\Sigma$ of operation symbols together with an arity mapping that assigns a natural $\operatorname{ar}(f)$ to every $f \in \Sigma$. If $\operatorname{ar}(f)$ is $0, f$ is called a constant.

For a set of variables Var, ranged over by $x, y, \ldots$, the set of (open) terms $\mathrm{T}(\Sigma, \operatorname{Var})$ over $\Sigma$ and Var, ranged over by $s, t, \ldots$, is the least set such that: 1$)$ each variable $x \in$ Var is a term; 2$) f\left(t_{1}, \ldots, t_{a r(f)}\right)$ is a term whenever $f \in \Sigma$ and $t_{1}, \ldots, t_{a r(f)}$ are terms. Closed terms are terms that do not contain variables.

A substitution is a mapping $\sigma: \operatorname{Var} \rightarrow \mathrm{T}(\Sigma$, Var $)$. With $\sigma(t)$ we denote the term obtained by replacing all occurrences of variables $x$ in term $t$ by $\sigma(x)$.

The abstract syntax of probabilistic process description languages is usually given by a signature $\Sigma$, whose closed terms are called probabilistic processes. The semantics is usually given by a PTS, where states are probabilistic processes. 


\section{Definitions}

In this section we introduce the notions of $\mathrm{PB}$ transition rule and $\mathrm{PB}$ transition system specification (PB stays for probabilistic bisimulation).

Definition 5. For any operation $f \in \Sigma$ and tuple $\vec{x}=x_{1}, \ldots, x_{a r(f)}$ of variables, a PB transition rule $\rho$ is of the form

$$
\begin{gathered}
\left\{x_{i} \stackrel{a_{i}, p_{i}}{\longrightarrow} y_{i} \mid i \in I\right\} \cup\left\{x_{j} \stackrel{A_{j}, p_{j}^{\prime}}{\longrightarrow} \mid j \in J\right\} \cup\left\{x_{h} \stackrel{B_{h}}{\longrightarrow} \mid h \in H\right\} \\
f(\vec{x}) \stackrel{a, \frac{\prod_{i \in I} p_{i}}{\prod_{j \in J}\left(1-p_{j}^{\prime}\right)} \cdot w_{\rho}}{\longrightarrow} t
\end{gathered}
$$

where:

1. $I, J, H$ are subsets of $\{1, \ldots$, ar $(f)\}$ such that $J \subseteq I$;

2. $a_{i} \in$ Act for $i \in I, A_{j} \subseteq$ Act for $j \in J, B_{h} \subseteq$ Act for $h \in H, a \in$ Act;

3. for all $i \in I$ and $j \in J$ such that $i=j$, it holds that $a_{i} \notin A_{j}$;

4. $p_{i}$ is a variable with range $(0,1]$ for $i \in I, p_{j}^{\prime}$ is a variable with range $[0,1)$ for $j \in J$;

5. $t$ is a term over $\Sigma$ and $\vec{x} \cup\left\{y_{i} \mid i \in I\right\}$;

6. $w_{\rho}$ is the weight of $\rho$ and satisfies $0<w_{\rho} \leq 1$.

Transitions $\left\{x_{i} \stackrel{a_{i}, p_{i}}{\longrightarrow} y_{i} \mid i \in I\right\}$ are the active premises; variables $\left\{x_{i} \mid i \in\right.$ $I\}$ are the active variables; transitions $\left\{x_{j} \stackrel{A_{j}, p_{j}^{\prime}}{\longrightarrow} \mid j \in J\right\}$ are the unneeded premises; transitions $\left\{x_{h} \stackrel{B_{h}}{\longrightarrow} \mid h \in H\right\}$ are the unquantified premises; transition $f(\vec{x}) \stackrel{a, \frac{\prod_{i \in I} p_{i}}{\prod_{j \in J}\left(1-p_{j}^{\prime}\right)} \cdot w_{\rho}}{\longrightarrow} t$ is the conclusion; $f(\vec{x})$ is the source; $t$ is the target of $\rho$.

Given terms $\vec{t}$, values $\left\{q_{i} \mid i \in I\right\}$ in $(0,1]$, and values $\left\{q_{j}^{\prime} \mid j \in J\right\}$ in $[0,1)$, Def. [5 says that term $f(\vec{t})$ has the move $f(\vec{t}) \stackrel{a, q}{\longrightarrow} t[\vec{t} / \vec{x}][\vec{s} / \vec{y}]$, with $q=$ $\frac{\prod_{i \in I} q_{i}}{\prod_{j \in J}\left(1-q_{j}^{\prime}\right)} \cdot w_{\rho}$, provided that $t_{i}$ has the move $t_{i} \stackrel{a_{i}, q_{i}}{\longrightarrow} s_{i}$, for all $i \in I$, the sum of the probability of the moves of $t_{j}$ with label in $A_{j}$ is $q_{j}^{\prime}$, for all $j \in J$, and $t_{h}$ has at least one move with label in $B_{h}$, for all $h \in H$.

Notice that the conclusion is triggered by both active and unquantified premises, and does not require unneeded premises, meaning that $p_{j}^{\prime}$ could be 0 for some $j \in J$. Unneeded premises are used to compute the probability of the conclusion. More precisely, they permit normalization of probability, which, as we will see in next sections, is needed in several operations of process algebras, such as restriction and priority. The probability of the conclusion depends on the weight of $\rho$ and on $\frac{\prod_{i \in I} p_{i}}{\prod_{j \in J}\left(1-p_{j}^{\prime}\right)}$, which is the conditional probability that all $x_{i}$ perform $a_{i}$ under the assumption that all $x_{j}$ are not allowed to perform actions in $A_{j}$. Unquantified premises do not contribute in computing the probability of the conclusion. They are "necessary conditions" for the application of $\rho$.

Definition 6. A PB transition system specification (PB TSS, for short) is formed by a set $\mathcal{R}$ of $P B$ transition rules such that: 
1. $\mathcal{R}$ is partitioned into $n$ strata $\mathcal{R}_{1}, \ldots, \mathcal{R}_{n}$, for some $n \in \mathbb{N}$;

2. for each stratum $\mathcal{R}_{u}$, operation $f$ and tuple of variables $\vec{x}=x_{1}, \ldots, x_{\text {ar }(f)}$ s.t. $\mathcal{R}_{u}$ has at least one PB transition rule with source $f(\vec{x})$, it holds that:

(a) All PB transition rules with source $f(\vec{x})$ in stratum $\mathcal{R}_{u}$ have the same set of unquantified premises $\left\{x_{h} \stackrel{B_{h}}{\longrightarrow} \mid h \in H\right\}$;

(b) All PB transition rules with source $f(\vec{x})$ in stratum $\mathcal{R}_{u}$ have the same set of unneeded premises $\left\{x_{j} \stackrel{A_{j}, p_{j}^{\prime}}{\longrightarrow} \mid j \in J\right\}$;

(c) All PB transition rules with source $f(\vec{x})$ in stratum $\mathcal{R}_{u}$ have the same set of active variables $\left\{x_{i} \mid i \in I\right\}$;

(d) Given actions $\left\{a_{i}^{\prime} \mid i \in I\right\}$ such that $a_{i}^{\prime} \notin A_{j}$ for all indexes $i$ and $j$ with $i=j$ and $x_{j} \stackrel{A_{j}, p_{j}^{\prime}}{\longrightarrow}$ an unneeded premise, then there is at least one $P B$ transition rule with source $f(\vec{x})$ in $\mathcal{R}_{u}$ with active premises $\left\{x_{i} \stackrel{a_{i}^{\prime}, p_{i}}{\longrightarrow} y_{i} \mid i \in I\right\}$

(e) Given the PB transition rules $\rho_{1}, \ldots, \rho_{m}$ in $\mathcal{R}_{u}$ with source $f(\vec{x})$ having the same active premises, their weights satisfy $w_{\rho_{1}}+\cdots+w_{\rho_{m}}=1$.

The meaning of clause 1 is that the rules in stratum $\mathcal{R}_{u}$ can be applied only if no rule in strata $\mathcal{R}_{1}, \ldots, \mathcal{R}_{u-1}$ can be applied (see Def. 7 below).

Let us take any $f \in \Sigma$. Clause $2 \mathrm{a}$ implies that unquantified premises trigger either all rules with source $f(\vec{x})$ in $\mathcal{R}_{u}$, or none of them. In the first case, we can prove that clauses $2 \mathrm{~b}$ 2e ensure that, given semistochastic processes $\vec{t}$, then the sum of the probability of the moves of $f(\vec{t})$ that are derivable by the rules in $\mathcal{R}_{u}$ is either 0 or 1 . Let us distinguish two cases. In the first case, some $t_{i}$ with $i \in I$ is not stochastic. Since it is semistochastic, $t_{i}$ has no move. Hence, since clause 2c implies that a move of $t_{i}$ is needed to infer a move of $f(\vec{t})$, no move of $f(\vec{t})$ can be derived from the rules in stratum $\mathcal{R}_{u}$, and, therefore, the sum of the probability of the moves of $f(\vec{t})$ derivable from $\mathcal{R}_{u}$ is 0 . In the second case, all $t_{i}$ with $i \in I$ are stochastic. Let us assume that, for all $j \in J, q_{j}^{\prime}$ is the probability such that $t_{j} \stackrel{A_{j}, q_{j}^{\prime}}{\longrightarrow}$. Value $\prod_{j \in J}\left(1-q_{j}^{\prime}\right)$ is the probability that each $t_{j}$ does not perform any action in $A_{j}$. All combinations of arbitrary moves $\left\{t_{i} \stackrel{a_{i}, q_{i}}{\longrightarrow} t_{i}^{\prime} \mid i \in I\right\}$, with $a_{i} \in$ Act for each $i \in I$, fall into two categories:

- Some $a_{i}$ is in $A_{j}$ for the index $j=i$. Clause 3 of Def. 5 ensures that no move of $f(\vec{t})$ is inferred by rules in $\mathcal{R}_{u}$ from moves $\left\{t_{i} \stackrel{a_{i}, q_{i}}{\longrightarrow} t_{i}^{\prime} \mid i \in I\right\}$.

- No $a_{i}$ is such that $a_{i} \in A_{j}$ for any index $j=i$. Since $t_{i}$ is semistochastic, this implies $q_{j}^{\prime} \neq 1$ for all $j \in J$. By clause 2d] of Def. [6] there exist rules $\rho_{1}, \ldots, \rho_{m}$ with source $f(\vec{x})$ in $\mathcal{R}_{u}$, for some $m \in \mathbb{N}$, with active premises $\left\{x_{i} \stackrel{a_{i}, p_{i}}{\longrightarrow} y_{i} \mid i \in I\right\}$. Hence, $f(\vec{t})$ has $m$ moves with probabilities $w_{\rho_{1}} \cdot \frac{\prod_{i \in I} q_{i}}{\prod_{j \in J}\left(1-q_{j}^{\prime}\right)}, \ldots, w_{\rho_{m}} \cdot \frac{\prod_{i \in I} q_{i}}{\prod_{j \in J}\left(1-q_{j}^{\prime}\right)}$. Notice that these probabilities are well defined, since $q_{j}^{\prime} \neq 1$ for all $j \in J$. Now, since $w_{\rho_{1}}+\cdots+w_{\rho_{m}}=1$ by clause 2e of Def. 6] the sum of these probabilities is $\frac{\prod_{i \in I} q_{i}}{\prod_{j \in J}\left(1-q_{j}^{\prime}\right)}$. 
Since we have assumed that all $\vec{t}$ are stochastic, and that for all $j \in J, q_{j}^{\prime}$ is the probability of $t_{j} \stackrel{A_{j}, q_{j}^{\prime}}{\longrightarrow}$, the overall probabilities of the combinations of moves $\left\{t_{i} \stackrel{a_{i}, q_{i}}{\longrightarrow} t_{i}^{\prime} \mid i \in I\right\}$ falling in the second category is $\prod_{j \in J}\left(1-q_{j}^{\prime}\right)$. Hence, if $q_{j}^{\prime}=1$ for some $j \in J, f(\vec{t})$ has no move and the sum of the probability of the moves of $f(\vec{t})$ derivable from $\mathcal{R}_{u}$ is 0 . Otherwise, if $q_{j}^{\prime} \neq 1$ for all $j \in J$, the sum of the probability of the moves of $f(\vec{t})$ derivable from $\mathcal{R}_{u}$ is $\frac{\prod_{j \in J}\left(1-q_{j}^{\prime}\right)}{\prod_{j \in J}\left(1-q_{j}^{\prime}\right)}=1$.

We can now formalize how PTSs are generated by PB TSSs.

Definition 7. Assume a PB TSS with strata $\mathcal{R}_{1}, \ldots, \mathcal{R}_{n}$.

1. A transition $t \stackrel{a, q}{\longrightarrow} s$ is provable from stratum $\mathcal{R}_{u}$ iff there is a closed substitution instance $\frac{\left\{t_{i} \stackrel{a_{i}, q_{i}}{\longrightarrow} s_{i} \mid i \in I\right\} \cup\left\{t_{j} \stackrel{A_{j}, q_{j}^{\prime}}{\longrightarrow} \mid j \in J\right\} \cup\left\{t_{h} \stackrel{B_{h}}{\longrightarrow} \mid h \in H\right\}}{t \stackrel{a, q}{\longrightarrow} s}$ of a PB transition rule in $\mathcal{R}_{u}$ such that:

(a) for all $i \in I, t_{i} \stackrel{a_{i}, q_{i}}{\longrightarrow} s_{i}$ is a transition provable from the TSS;

(b) for all $j \in J, q_{j}^{\prime}=\sum\left\{|q| \exists a \in A_{j}, s^{\prime}: t_{j} \stackrel{a, q}{\longrightarrow} s^{\prime}\right.$ is provable from the TSS $\left.\mid\right\}$;

(c) for all $h \in H$, at least one transition $t_{h} \stackrel{a, q_{h}}{\longrightarrow} u_{h}$ with $a \in B_{h}$ is provable from the TSS, for some $q_{h}$ and $u_{h}$;

2. A transition $t \stackrel{a, q}{\longrightarrow} s$ is provable from the TSS if it is provable from some stratum $\mathcal{R}_{u}$ and no transition with source $t$ is provable from strata $\mathcal{R}_{1}, \ldots, \mathcal{R}_{u-1}$.

Moves of terms are proved inductively w.r.t. their structure. In fact, first of all we can prove moves of constants from strata $\mathcal{R}_{1}, \ldots, \mathcal{R}_{n}$ and, then, we can prove moves of constants from the TSS. This is possible since PB transition rules having a constant as source have no premise. Then, after moves of terms $\vec{t}$ have been proved from the TSS, we can prove moves of $f(\vec{t})$ from $\mathcal{R}_{1}, \ldots, \mathcal{R}_{n}$ and, then, we can prove moves of $f(\vec{t})$ from the TSS.

Let us recall that, according to the classical definition (see, e.g., [12]), a (nonprobabilistic) transition $t \stackrel{a}{\rightarrow} t^{\prime}$ is provable from a given TSS iff there exists a wellfounded, upwardly branching tree whose nodes are labeled by closed transitions, whose leaves have empty label, whose root is labeled by $t \stackrel{a}{\rightarrow} t^{\prime}$, and, whenever $K$ is the (possibly empty) set of labels of the nodes directly above a node labeled by $\beta$, then $K / \beta$ is a closed substitution instance of a transition rule in the TSS.

We need a more complicated definition since our rules have the unneeded premises and the unquantified premises that are not "pure" transitions. Hence, we cannot construct the branching tree that is considered in the classical definition. Moreover, as in [19], we have to take into account that there is an ordering relation between the transition rules, given by the partitioning in $n$ strata.

Definition 8. The PTS induced by a PB TSS is the PTS having as transitions the transitions that are provable from the TSS. 


\section{Examples}

In this section we show that most of operations offered by the probabilistic process algebras proposed in the literature can be expressed by our PB TSSs.

Example 1 (Constants). Stratum $\mathcal{R}_{1}$ contains the following rule, for all $a \in$ Act:

$$
\overline{a \stackrel{a, 1}{\longrightarrow} 0}
$$

Term $a$ performs action $a$, and, then, it behaves as the idle process 0 .

Let us show now that we can express the probabilistic sum of $[2,3,8,9,11$.

Example 2 (Probabilistic sum). Let $0<p<1$. Stratum $\mathcal{R}_{1}$ contains the following rules, for all $a_{1}, a_{2} \in$ Act, where $p$ and $1-p$ are their weights:

$$
\stackrel{x_{1} \stackrel{a_{1}, p_{1}}{\longrightarrow} y_{1} \quad x_{2} \stackrel{a_{2}, p_{2}}{\longrightarrow} y_{2}}{x_{1}+{ }^{p} x_{2} \stackrel{a_{1}, p_{1} \cdot p_{2} \cdot p}{\longrightarrow} y_{1}} \quad \quad \frac{x_{1} \stackrel{a_{1}, p_{1}}{\longrightarrow} y_{1} \quad x_{2} \stackrel{a_{2}, p_{2}}{\longrightarrow} y_{2}}{x_{1}+{ }^{p} x_{2} \stackrel{a_{2}, p_{1} \cdot p_{2} \cdot(1-p)}{\longrightarrow} y_{2}}
$$

Stratum $\mathcal{R}_{2}$ contains the following rule, for all $a_{1} \in$ Act:

$$
\frac{x_{1} \stackrel{a_{1}, p_{1}}{\longrightarrow} y_{1}}{x_{1}+{ }^{p} x_{2} \stackrel{a_{1}, p_{1}}{\longrightarrow} y_{1}}
$$

Stratum $\mathcal{R}_{3}$ contains the following rule, for all $a_{2} \in$ Act:

$$
\frac{x_{2} \stackrel{a_{2}, p_{2}}{\longrightarrow} y_{2}}{x_{1}+{ }^{p} x_{2} \stackrel{a_{2}, p_{2}}{\longrightarrow} y_{2}}
$$

Let us take term $t_{1}+{ }^{p} t_{2}$. Index $p$ means that, when both $t_{1}$ and $t_{2}$ can move, $t_{1}$ moves with probability $p$, and $t_{2}$ moves with probability $1-p$. Rules in $\mathcal{R}_{1}$ (with weights $p$ and $1-p$ ) are applied when both $t_{1}$ and $t_{2}$ are stochastic; rules in $\mathcal{R}_{2}$ (with weight 1 ) are applied when only $t_{1}$ is stochastic; rules in $\mathcal{R}_{3}$ (with weight 1 ) are applied when only $t_{2}$ is stochastic. In the first case, since $t_{2}$ (resp. $t_{1}$ ) is stochastic and the sum of the probability of its moves is 1 , from $t_{1} \stackrel{a_{1}, p_{1}}{\longrightarrow} t_{1}^{\prime}$ (resp. $t_{2} \stackrel{a_{2}, p_{2}}{\longrightarrow} t_{2}^{\prime}$ ) we infer moves of $t_{1}+^{p} t_{2}$ labeled $a_{1}$ (resp. $a_{2}$ ) with total probability $p_{1} \cdot p$ (resp. $\left.p_{2} \cdot(1-p)\right)$. In the other two cases, from $t_{1} \stackrel{a_{1}, p_{1}}{\longrightarrow} t_{1}^{\prime}$ (resp. $t_{2} \stackrel{a_{2}, p_{2}}{\longrightarrow} t_{2}^{\prime}$ ), we infer $t_{1}+^{p} t_{2} \stackrel{a_{1}, p_{1}}{\longrightarrow} t_{1}^{\prime}$ (resp. $t_{1}+^{p} t_{2} \stackrel{a_{2}, p_{2}}{\longrightarrow} t_{2}^{\prime}$ ).

Let us consider now the interleaving operation of [3].

Example 3 (Interleaving). Let $0<p<1$. Stratum $\mathcal{R}_{1}$ contains the following rules, for all $a_{1}, a_{2} \in$ Act, where $p$ and $1-p$ are their weights:

$$
\frac{x_{1} \stackrel{a_{1}, p_{1}}{\longrightarrow} y_{1} \quad x_{2} \stackrel{a_{2}, p_{2}}{\longrightarrow} y_{2}}{x_{1}\left\|^{p} x_{2} \stackrel{a_{1}, p_{1} \cdot p_{2} \cdot p}{\longrightarrow} y_{1}\right\|^{p} x_{2}} \quad \frac{x_{1} \stackrel{a_{1}, p_{1}}{\longrightarrow} y_{1} \quad x_{2} \stackrel{a_{2}, p_{2}}{\longrightarrow} y_{2}}{x_{1}\left\|^{p} x_{2} \stackrel{a_{2}, p_{1} \cdot p_{2} \cdot(1-p)}{\longrightarrow} x_{1}\right\|^{p} y_{2}}
$$


Stratum $\mathcal{R}_{2}$ contains the following rules, for all $a_{1} \in$ Act:

$$
\frac{x_{1} \stackrel{a_{1}, p_{1}}{\longrightarrow} y_{1}}{x_{1}\left\|^{p} x_{2} \stackrel{a_{1}, p_{1}}{\longrightarrow} y_{1}\right\|^{p} x_{2}}
$$

Stratum $\mathcal{R}_{3}$ contains the following rules, for all $a_{2} \in$ Act:

$$
\frac{x_{2} \stackrel{a_{2}, p_{2}}{\longrightarrow} y_{2}}{x_{1}\left\|^{p} x_{2} \stackrel{a_{2}, p_{2}}{\longrightarrow} x_{1}\right\|^{p} y_{2}}
$$

As in Ex. 2, given a term $t_{1} \|^{p} t_{2}$, index $p$ means that, when both $t_{1}$ and $t_{2}$ can move, $t_{1}$ moves with probability $p$, and $t_{2}$ moves with probability $1-p$.

Let us consider now the synchronous product of PCCS [10, 11].

Example 4 (Synchronous product). Stratum $\mathcal{R}_{1}$ contains the following rules, for all $a_{1}, a_{2} \in$ Act:

$$
\frac{x_{1} \stackrel{a_{1}, p_{1}}{\longrightarrow} y_{1} \quad x_{2} \stackrel{a_{2}, p_{2}}{\longrightarrow} y_{2}}{x_{1}\left\|x_{2} \stackrel{a_{1} \times a_{2}, p_{1} \cdot p_{2}}{\longrightarrow} y_{1}\right\| y_{2}}
$$

Here, at each computation step, term $t_{1} \| t_{2}$ can move only by combining an action of $t_{1}$ and an action of $t_{2}$. Actions are composed by means of operator $\times$.

Let us consider now the probabilistic version of CCS parallel composition [3].

Example 5 (Interleaving plus synchronization). Let $0<p, q<1$. Stratum $\mathcal{R}_{1}$ contains the following rules, for all $a_{1}, a_{2} \in$ Act such that $a_{2} \neq \overline{a_{1}}$ :

$$
\begin{array}{cc}
\frac{x_{1} \stackrel{a_{1}, p_{1}}{\longrightarrow} y_{1} \quad x_{2} \stackrel{a_{2}, p_{2}}{\longrightarrow} y_{2}}{x_{1}\left\|_{q}^{p} x_{2} \stackrel{a_{1}, p_{1} \cdot p_{2} \cdot p}{\longrightarrow} y_{1}\right\|_{q}^{p} x_{2}} & \frac{x_{1} \stackrel{a_{1}, p_{1}}{\longrightarrow} y_{1} x_{2} \stackrel{a_{2}, p_{2}}{\longrightarrow} y_{2}}{x_{1}\left\|_{q}^{p} x_{2} \stackrel{a_{2}, p_{1} \cdot p_{2} \cdot(1-p)}{\longrightarrow} x_{1}\right\|_{q}^{p} y_{2}} \\
\stackrel{x_{1} \stackrel{a_{1}, p_{1}}{\longrightarrow} y_{1} \quad x_{2} \stackrel{\overline{a_{1}}, p_{2}}{\longrightarrow} y_{2}}{x_{1}\left\|_{q}^{p} x_{2} \stackrel{a_{1}, p_{1} \cdot p_{2} \cdot p \cdot(1-q)}{\longrightarrow} y_{1}\right\|_{q}^{p} x_{2}} & \frac{x_{1} \stackrel{a_{1}, p_{1}}{\longrightarrow} y_{1} x_{2} \stackrel{\overline{a_{1}}, p_{2}}{\longrightarrow} y_{2}}{x_{1}\left\|_{q}^{p} x_{2} \stackrel{\overline{a_{1}}, p_{1} \cdot p_{2} \cdot(1-p) \cdot(1-q)}{\longrightarrow} x_{1}\right\|_{q}^{p} y_{2}} \\
\frac{x_{1} \stackrel{a_{1}, p_{1}}{\longrightarrow} y_{1} \frac{x_{2} \stackrel{\overline{a_{1}}, p_{2}}{\longrightarrow} y_{2}}{x_{1}\left\|_{q}^{p} x_{2} \stackrel{\tau, p_{1} \cdot p_{2} \cdot q}{\longrightarrow} y_{1}\right\|_{q}^{p} y_{2}}}{}
\end{array}
$$

Stratum $\mathcal{R}_{2}$ contains the following rules, for all $a_{1} \in$ Act:

$$
\frac{x_{1} \stackrel{a_{1}, p_{1}}{\longrightarrow} y_{1}}{x_{1}\left\|_{q}^{p} x_{2} \stackrel{a_{1}, p_{1}}{\longrightarrow} y_{1}\right\|_{q}^{p} x_{2}}
$$

Stratum $\mathcal{R}_{3}$ contains the following rules, for all $a_{2} \in$ Act:

$$
\frac{x_{2} \stackrel{a_{2}, p_{2}}{\longrightarrow} y_{2}}{x_{1}\left\|_{q}^{p} x_{2} \stackrel{a_{2}, p_{2}}{\longrightarrow} x_{1}\right\|_{q}^{p} y_{2}}
$$


Let us take $t_{1} \|_{q}^{p} t_{2}$. When $t_{1}$ and $t_{2}$ intend to perform actions $a_{1}$ and $a_{2}$ with $a_{2} \neq \overline{a_{1}}, t_{1}$ moves with probability $p$ and $t_{2}$ moves with probability $1-p$, as in the case of interleaving operator of Ex. 3. When $t_{1}$ and $t_{2}$ intend to perform actions $a_{1}$ and $\overline{a_{1}}$, either they synchronize with probability $q$, thus producing action $\tau$, or they do not synchronize with probability $1-q$. In this second case, $t_{1}$ moves with probability $p \cdot(1-q)$, and $t_{2}$ moves with probability $(1-p) \cdot(1-q)$.

Let us consider now the operation of sequential composition of terms of [3]. Example 6 (Sequencing). Stratum $\mathcal{R}_{1}$ contains the following rules, for $a_{1} \in$ Act:

$$
\frac{x_{1} \stackrel{a_{1}, p_{1}}{\longrightarrow} y_{1}}{x_{1} \cdot x_{2} \stackrel{a_{1}, p_{1}}{\longrightarrow} y_{1} \cdot x_{2}}
$$

Stratum $\mathcal{R}_{2}$ contains the following transition rules, for all $a_{2} \in$ Act:

$$
\frac{x_{2} \stackrel{a_{2}, p_{2}}{\longrightarrow} y_{2}}{x_{1} \cdot x_{2} \stackrel{a_{2}, p_{2}}{\longrightarrow} y_{2}}
$$

Let us take $t_{1} \cdot t_{2}$. If $t_{1}$ moves, then rules in $\mathcal{R}_{1}$ can be applied and $t_{1} \cdot t_{2}$ moves as $t_{1}$, else, if $t_{2}$ moves, rules in $\mathcal{R}_{2}$ can be applied and $t_{1} \cdot t_{2}$ moves as $t_{2}$.

Let us consider now the restriction operation of [2, 8, 9, 11. This is the first example in which we employ unneeded premises.

Example 7 (Restriction). Let $A \subseteq$ Act. Stratum $\mathcal{R}_{1}$ contains the following rules, for all $a_{1} \in$ Act $\backslash A$ :

$$
\frac{x_{1} \stackrel{a_{1}, p_{1}}{\longrightarrow} y_{1} \quad x_{1} \stackrel{A, p}{\longrightarrow}}{x_{1} \backslash A \stackrel{a_{1}, \frac{p_{1}}{1-p}}{\longrightarrow} y_{1} \backslash A}
$$

Term $t_{1} \backslash A$ behaves as $t_{1}$, but it cannot perform actions in $A$. Let us assume that the sum of the probability of the moves of $t_{1}$ with label in $A$ is $q$, i.e. $t_{1} \stackrel{A, q}{\longrightarrow}$. If $q=1$, then no move of $t_{1} \backslash A$ can be inferred by the rules in $\mathcal{R}_{1}$. Hence, $t_{1} \backslash A$ has no move and it is semistochastic. If $t_{1}$ has a move $t_{1} \stackrel{a_{1}, q_{1}}{\longrightarrow} t_{1}^{\prime}$, with $a_{1} \notin A$, then $t_{1} \backslash A$ has the same move, but with probability $\frac{q_{1}}{1-q}$, which is the conditional probability that $t_{1}$ has the move $t_{1} \stackrel{a_{1}, q_{1}}{\longrightarrow} t_{1}^{\prime}$ under the assumption that $t_{1}$ is not allowed to perform actions in $A$. Hence, the sum of the probability of the moves of $t_{1} \backslash A$ is $\frac{1-q}{1-q}=1$, and $t_{1} \backslash A$ is stochastic.

Let us consider now the operator of priority. This is the first example in which we employ unquantified premises.

Example 8 (Priority of a over $b$ ). Let $a, b \in$ Act. Stratum $\mathcal{R}_{1}$ contains the following rules, for all $a_{1} \in A c t \backslash\{b\}$ :

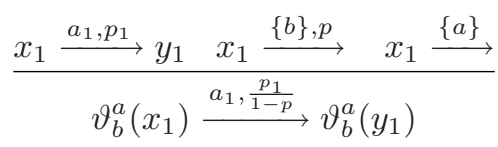


Stratum $\mathcal{R}_{2}$ contains the following rules, for all $a_{1} \in$ Act:

$$
\frac{x_{1} \stackrel{a_{1}, p_{1}}{\longrightarrow} y_{1}}{\vartheta_{b}^{a}\left(x_{1}\right) \stackrel{a_{1}, p_{1}}{\longrightarrow} \vartheta_{b}^{a}\left(y_{1}\right)}
$$

Term $\vartheta_{b}^{a}\left(t_{1}\right)$ behaves as $t_{1}$, but it can perform action $b$ only if it cannot perform $a$. Rules in $\mathcal{R}_{1}$ are applied only if $t_{1}$ can perform $a$. In this case, if the sum of the probability of the moves of $t_{1}$ labeled $b$ is $q$ (i.e. $t_{1} \stackrel{\{b\}, q}{\longrightarrow}$ ), then, from any move $t_{1} \stackrel{a_{1}, q_{1}}{\longrightarrow} t_{1}^{\prime}$ with $a_{1} \neq b$, we infer a move of $\vartheta_{b}^{a}\left(t_{1}\right)$ with label $a_{1}$ and probability $\frac{q_{1}}{1-q}$, which is the conditional probability that $t_{1}$ has the move $t_{1} \stackrel{a_{1}, q_{1}}{\longrightarrow} t_{1}^{\prime}$ under the assumption that $t_{1}$ is not allowed to perform $b$. So, the sum of the probability of the moves of $\vartheta_{b}^{a}\left(t_{1}\right)$ is $\frac{1-q}{1-q}=1$, and $\vartheta_{b}^{a}\left(t_{1}\right)$ is stochastic. Rules in $\mathcal{R}_{2}$ can be applied only if $t_{1}$ cannot perform $a$. In this case, $\vartheta_{b}^{a}\left(t_{1}\right)$ behaves as $t_{1}$.

\section{Results}

Theorem 1. The PTS induced by any PB TSS is semistochastic.

Proof. We have to prove that, given an arbitrary term $t$, the sum of the probability of the moves of $t$ is either 0 or 1 . This property follows by two facts: 1) The moves of $t$ can be derived only by the rules that are in one stratum $\mathcal{R}_{u} ; 2$ ) the sum of the probability of the moves of $t$ derivable by the rules in any stratum $\mathcal{R}_{u}$ is either 0 or 1 , as we have proved in the previous section.

Theorem 2. The probabilistic bisimulation induced by any PB TSS is a congruence.

Proof. Let $R$ be the least equivalence relation over PTS states such that:

1. $s R t$ whenever $s \approx t$;

2. $f(\vec{s}) R f(\vec{t})$ whenever $s_{1} R t_{1}, \ldots, s_{a r(f)} R t_{a r(f)}$.

Lemma 1. Given a term $u$ over variables $\vec{x}=x_{1}, \ldots, x_{n}$ and tuples of terms $\vec{s}=s_{1}, \ldots, s_{n}$ and $\vec{t}=t_{1}, \ldots, t_{n}$, if $s_{i} R t_{i}$ holds for all $1 \leq i \leq n$, then $u[\vec{t} / \vec{x}] R u[\vec{s} / \vec{x}]$.

To prove the thesis, it suffices to prove that, for arbitrary terms $s$ and $t, s R t$ implies $s \approx t$. In fact, by the two clauses of the definition of $R$, this property implies that $R$ and $\approx$ coincide and that $\approx$ is a congruence.

Let us reason by induction over the definition of $R$. The base case where $s R t$ is due to $s \approx t$ is immediate. Let us concentrate on the inductive step, where $s \equiv f(\vec{s}), t \equiv f(\vec{t})$, and $s R t$ is due to $s_{1} R t_{1}, \ldots, s_{a r(f)} R t_{a r(f)}$. We can assume, by the inductive hypothesis, that $s_{1} \approx t_{1}, \ldots, s_{a r(f)} \approx t_{a r(f)}$. 
We have to prove that, for any value $0<q \leq 1$, action $a \in$ Act and equivalence class $S \in \mathcal{S} / R, \mu(f(\vec{s}), a, S)=q$ iff $\mu(f(\vec{t}), a, S)=q$. We prove that $\mu(f(\vec{s}), a, S)=q$ implies $\mu(f(\vec{t}), a, S)=q$; the converse is analogous.

Since $\mu(f(\vec{s}), a, S)=q$, it holds that in some stratum $\mathcal{R}_{u}$ of the TSS, and for some $k \in \mathbb{N}$, there exist $\mathrm{PB}$ transition rules $\rho_{1}, \ldots, \rho_{k}$ such that:

1. for all $1 \leq l \leq k$, from rule $\rho_{l}$ we infer $m_{l}$ transitions $f(\vec{s}) \stackrel{a, q_{l, 1}}{\longrightarrow} u_{l, 1}, \ldots$, $f(\vec{s}) \stackrel{a, q_{l, m_{l}}}{\longrightarrow} u_{l, m_{l}}$, for some $m_{l} \in \mathbb{N}$;

2. $\sum_{1 \leq l \leq k} \sum_{1 \leq i \leq m_{l}} q_{l, i}=q$;

3. for all $1 \leq l \leq k, u_{l, 1}, \ldots, u_{l, m_{l}} \in S$,

and, moreover, no move of $f(\vec{s})$ is derived from rules in $\mathcal{R}_{1}, \ldots, \mathcal{R}_{u-1}$.

Let us consider any $1 \leq l \leq k$. Transition rule $\rho_{l}$ has the form

$$
\frac{\left\{x_{i} \stackrel{a_{i}, p_{i}}{\longrightarrow} y_{i} \mid i \in I\right\} \cup\left\{x_{j} \stackrel{A_{j}, p_{j}^{\prime}}{\longrightarrow} \mid j \in J\right\} \cup\left\{x_{h} \stackrel{B_{h}}{\longrightarrow} \mid h \in H\right\}}{f(\vec{x}) \stackrel{a, \frac{\prod_{i \in I} p_{i}}{\prod_{j \in J}\left(1-p_{j}^{\prime}\right)} \cdot w_{\rho_{l}}}{\longrightarrow} t}
$$

Since $f(\vec{s}) \stackrel{a, q_{l, 1}}{\longrightarrow} u_{l, 1}, \ldots, f(\vec{s}) \stackrel{a, q_{l, m_{l}}}{\longrightarrow} u_{l, m_{l}}$ are derived from $\rho_{l}$, it holds that:

1. for all $i \in I$, there are states $S_{i}$ s.t. $\mu\left(s_{i}, a_{i}, S_{i}\right)=q_{i}$, for some $0<q_{i} \leq 1$;

2. for all $j \in J, s_{j} \stackrel{A_{j}, q_{j}^{\prime}}{\longrightarrow}$, for some $0 \leq q_{j}^{\prime}<1$;

3. for all $h \in H, s_{h} \stackrel{B_{h}}{\longrightarrow}$;

4. $q_{l, 1}+\cdots+q_{l, m_{l}}=w_{\rho_{l}} \cdot \frac{\prod_{i \in I} q_{i}}{\prod_{j \in J}\left(1-q_{j}^{\prime}\right)}$.

By the inductive hypothesis, it follows that:

1. for all $i \in I$, there is a set of states $S_{i}^{\prime}$ such that $\mu\left(t_{i}, a_{i}, S_{i}^{\prime}\right)=q_{i}$ and, for all $s^{\prime} \in S_{i}^{\prime}$, there is some state $s \in S_{i}$ such that $s R s^{\prime}$;

2. for all $j \in J, t_{j} \stackrel{A_{j}, q_{j}^{\prime}}{\longrightarrow}$;

3. for all $h \in H, t_{h} \stackrel{B_{h}}{\longrightarrow}$.

Hence, by applying $\rho_{l}$, we infer $n_{l}$ moves $f(\vec{t}) \stackrel{a, q_{l, 1}^{\prime}}{\longrightarrow} v_{1}, \ldots f(\vec{t}) \stackrel{a, q_{l, n_{l}}^{\prime}}{\longrightarrow} v_{n_{l}}$, for some $n_{l} \in \mathbb{N}$, where:

1. $v_{1}, \ldots, v_{n_{l}} \in S$, by Lemma 1 and the fact that for all $s^{\prime} \in S_{i}^{\prime}$ there is some state $s \in S_{i}$ such that $s R s^{\prime}$;

2. $q_{l, 1}^{\prime}+\cdots+q_{l, n_{l}}^{\prime}=q_{l, 1}+\cdots+q_{l, m_{l}}$.

Since these arguments hold for all $1 \leq l \leq k$, it follows that by $\rho_{1}, \ldots, \rho_{k}$ we derive $\mu(f(\vec{t}), a, S)=q$, which implies the thesis. It remains to prove that we can apply $\rho_{1}, \ldots, \rho_{k}$, i.e. no move of $f(\vec{t})$ can be derived by any rule in any stratum $\mathcal{R}_{v}$ with $v<u$. This follows by the fact that no move of $f(\vec{s})$ can be derived by any rule in these strata, and that $s_{i} \approx t_{i}$ for $1 \leq i \leq \operatorname{ar}(f)$. 


\section{$6 \quad$ Extensions}

The PB transition rules of Def. 5 extend the rules matching the de Simone format [16] with probability, unneeded premises and unquantified premises. Here we show how we can add to our rules some features offered by other formats proposed in the literature of non probabilistic process algebras.

The GSOS format [7] admits negative premises of the form $x_{i} \stackrel{q_{i}}{\longrightarrow}$ in rules with source $f(\vec{x})$, meaning that the $i^{t h}$ argument of $f$ does not perform any action labeled $a_{i}$. In [19] a result is proved which assesses that negative premises can be simulated by suitable ordering relations between rules. Since the partitioning in strata of Def. [6 introduces ordering relations between PB transition rules that are less general than those used in [19, it would be interesting to extend Def. [6 to capture all the ordering relations of [19].

The GSOS format admits also double testing. Namely, rules with source $f(\vec{x})$ can have two (or more) premises $x_{i} \stackrel{a_{i_{1}}}{\longrightarrow} y_{i_{1}}$ and $x_{i} \stackrel{a_{i_{2}}}{\longrightarrow} y_{i_{2}}$ with the same variable $x_{i}$ in the left side. Let us show how we can add double testing to our rules.

Definition 9. A PB transition rule with double testing $\rho$ is of the form

$$
\frac{\left\{x_{i} \stackrel{a_{i_{l}}, p_{i_{l}}}{\longrightarrow} y_{i_{l}} \mid i \in I, l \in I_{i}\right\} \cup\left\{x_{j} \stackrel{A_{j}, p_{j}^{\prime}}{\longrightarrow} \mid j \in J\right\} \cup\left\{x_{h} \stackrel{B_{h}}{\longrightarrow} \mid h \in H\right\}}{f(\vec{x}) \stackrel{a, \frac{\prod_{i \in I} \sum_{l \in I_{i}} p_{i_{l}}}{\prod_{j \in J}\left(1-p_{j}^{\prime}\right)} \cdot w_{\rho}}{\longrightarrow} t}
$$

where:

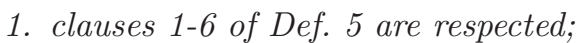

2. for all $i \in I$, it holds that $a_{i_{l}} \neq a_{i_{l^{\prime}}}$ for all $l, l^{\prime} \in I_{i}$ such that $l \neq l^{\prime}$;

3. for all $i \in I$ and $l \in I_{i}$, if $\left|I_{i}\right|>1$ then there is an $h=i$ such that $a_{i_{l}} \in B_{h}$.

Definition 10. A PB TSS with double testing is defined as in Def. 6. except that clause $2 \mathrm{Dd}$ is replaced by the following clause:

- Given actions $\left\{a_{i}^{\prime} \mid i \in I\right\}$ such that $a_{i}^{\prime} \notin A_{j}$ for all indexes $i$ and $j$ with $i=j$ and $x_{j} \stackrel{A_{j}, p_{j}^{\prime}}{\longrightarrow}$ an unneeded premise, then there at least one $P B$ transition rule with source $f(\vec{x})$ in $\mathcal{R}_{u}$ containing the active premises $\left\{x_{i} \stackrel{a_{i}^{\prime}, p_{i}}{\longrightarrow} y_{i} \mid i \in I\right\}$.

To explain clause 2 in Def. 9, let us take the following rule, which violates it:

$$
\underset{f\left(x_{1}\right) \stackrel{x_{1} \stackrel{a, p_{1}+p_{2}}{\longrightarrow} 0}{\stackrel{x_{1}}{\longrightarrow}} y_{2}}{\stackrel{a, p_{2}}{\longrightarrow}}
$$

Let $t$ be the PCCS term $a \cdot 0$, which has the move $t \stackrel{a, 1}{\longrightarrow} 0$. It holds that $f(t) \stackrel{b, 2}{\longrightarrow} 0$, and, therefore, $f(t)$ is not semistochastic. The problem is that the probability of the same move of $t$ is summed twice when computing the probability of the 
move of $f(t)$. Clause 2 in Def. 9 prevents this problem, since different moves of the same argument of $f$ can appear as premises only if they have different labels.

To explain clause 3 in Def. 9] let us take the following rules, and note that the first one violates it:

$$
\frac{x_{1} \stackrel{a, p_{1}}{\longrightarrow} y_{1} x_{1} \stackrel{b, p_{2}}{\longrightarrow} y_{2}}{f\left(x_{1}\right) \stackrel{d, p_{1}+p_{2}}{\longrightarrow} 0} \quad \frac{x_{1} \stackrel{c, p_{1}}{\longrightarrow} y_{1}}{f\left(x_{1}\right) \stackrel{e, p_{1}}{\longrightarrow} 0}
$$

Let $t$ be the PCCS term $a \cdot 0+^{\frac{1}{2}} c \cdot 0$, which has the moves $t \stackrel{a, \frac{1}{2}}{\longrightarrow} 0$ and $t \stackrel{c, \frac{1}{2}}{\longrightarrow}$ 0 . It holds that $f(t) \stackrel{e, \frac{1}{2}}{\longrightarrow} 0$ is the only move of $f(t)$, which, therefore, is not semistochastic. The problem is that the probability of the move of $t$ labeled $a$ does not contribute in computing the probability of any move of $f(t)$, since $t$ has no move labeled $b$ and the premise $x_{1} \stackrel{a, p_{1}}{\longrightarrow} y_{1}$ appears only in the rule where there is also the premise $x_{1} \stackrel{b, p_{2}}{\longrightarrow} y_{2}$. Clause 3 in Def. 9 prevents this problem, since premises $x_{1} \stackrel{a, p_{1}}{\longrightarrow} y_{1}$ and $x_{1} \stackrel{b, p_{2}}{\longrightarrow} y_{2}$ are admitted only in rules that are in strata where all rules have an unquantified premise $x_{1} \stackrel{B}{\longrightarrow}$ with $a, b \in B$.

Finally, notice that the new clause of Def. 10 requires that at least one rule in $\mathcal{R}_{u}$ contains the premises $\left\{x_{i} \stackrel{a_{i}^{\prime}, p_{i}}{\longrightarrow} y_{i} \mid i \in I\right\}$, whereas the corresponding clause in Def. 6 requires that at least one rule in $\mathcal{R}_{u}$ has exactly the premises $\left\{x_{i} \stackrel{a_{i}^{\prime}, p_{i}}{\longrightarrow} y_{i} \mid i \in I\right\}$. The new clause allows double testing.

Theorem 3. The PTS induced by any PB TSS with double testing is semistochastic. The probabilistic bisimulation induced by any PB TSS with double testing is a congruence.

The tyxt/tyft format [12] admits look ahead. Namely, transition rules with source $f(\vec{x})$ can have premises $x_{i} \stackrel{a_{i}}{\longrightarrow} y_{i}$ and $y_{i} \stackrel{b_{i}}{\longrightarrow} z_{i}$, with the same variable $y_{i}$ appearing in the right side of the first premise and in the left side of the second premise. Let us show how we can add look ahead to our PB TSSs.

Definition 11. A PB transition rule with look ahead $\rho$ is of the form

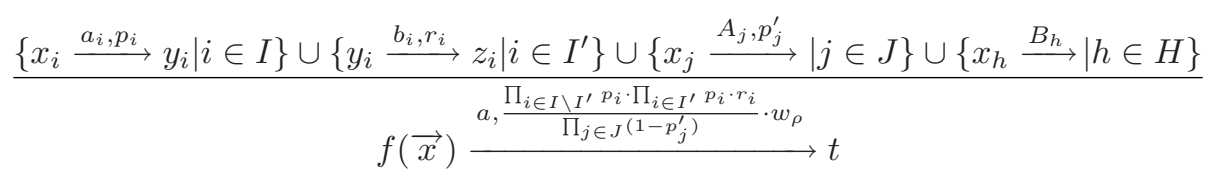

where:

1. clauses [1] 6 of Def. 5 are respected;

2. $I^{\prime} \subseteq I$.

Also variables $y_{i}$ with $i \in I^{\prime}$ are called active variables.

Definition 12. A PB TSS with look ahead is defined as in Def. 6, except that clauses 2C and 2d are replaced by the following clauses: 
1. All PB transition rules with source $f(\vec{x})$ in stratum $\mathcal{R}_{u}$ have the same set of active variables $\left\{x_{i} \mid i \in I\right\} \cup\left\{y_{i} \mid i \in I^{\prime}\right\}$;

2. Given actions $\left\{a_{i}^{\prime} \mid i \in I\right\}$ such that $a_{i}^{\prime} \notin A_{j}$ for all indexes $i$ and $j$ with $i=j$ and $x_{j} \stackrel{A_{j}, p_{j}^{\prime}}{\longrightarrow}$ an unneeded premise, and actions $b_{i}^{\prime}$ for all indexes $i \in I^{\prime}$, then there is at least one PB transition rule with source $f(\vec{x})$ in $\mathcal{R}_{u}$ with active premises $\left\{x_{i} \stackrel{a_{i}^{\prime}, p_{i}}{\longrightarrow} y_{i} \mid i \in I\right\} \cup\left\{y_{i} \stackrel{b_{i}^{\prime}, r_{i}}{\longrightarrow} z_{i} \mid i \in I^{\prime}\right\}$.

The new clauses in Deff. 11,12 extend clauses in Deff. 5 to take into account that two consecutive moves of $x_{i}$ are considered for all $i \in I^{\prime}$.

Theorem 4. The PTS induced by any PB TSS with look ahead is semistochastic. The probabilistic bisimulation induced by any PB TSS with look ahead is a congruence.

Definitions of PB transition rule and PB TSS admitting both double testing and look ahead could be given immediately. By combining results of Thm. 3 and Thm. 4 we infer that the PB TSSs so obtained would induce semistochastic PTSs and probabilistic bisimulations being congruences.

Both path format [4] and panth format [20] admit predicates, i.e. transitions of the form $t P$, meaning that term $t$ satisfies some property expressed by $P$. Since predicates have nothing to do with probability, they can be added to PB transitions rules and PB TSSs, without affecting results in Thm. 1 and Thm. 2

\section{Related and Future Work}

In this paper we have proposed a rule format for probabilistic process algebras. We believe that our format has four main merits: 1) probabilistic bisimulation is a congruence w.r.t. process algebra operations respecting the format; 2) semistochasticity is preserved by process algebra operations respecting the format; 3) the main operations offered by the probabilistic process algebras studied in the literature are captured by the format, which, therefore, has practical applications; 4) features offered by known rule formats proposed for classic process algebras, such as look ahead and double testing, are offered by the format.

Now, let us recall that in [6] a rule format for probabilistic process algebras has been already proposed. The first difference between our paper and [6] is that we consider the generative model of probabilistic processes, whereas [6] considers the reactive model. Then, our definition of TSS requires some conditions (i.e. clauses 2c 2e in Def. 6) that guarantee semistochasticity. In [6] no syntactic constraint on transition rules guarantees semistochasticity of reactive processes, i.e. the property that the sum of the probability of the moves of any process for the same label is either 0 or 1 . Hence, in [6] semistochasticity is not ensured by the format. In [6] neither unquantified premises nor unneeded premises nor stratification are considered. We need these features to express operations requiring redistribution of probability, such as restriction (see Ex. 7) and priority (see Ex. 
8). In the reactive model restriction and priority do not require redistribution of probability, and, therefore, they can be expressed with the format in 6]. Problems in [6] arise in other operations requiring redistribution of probability, such as the relabeling operation $t[f]$, where $f: A c t \longrightarrow$ Act is a relabeling functions.

Our results can be extended in several directions. We aim to develop a rule format for the reactive model of probabilistic processes that guarantees results analogous to those obtained in the present paper, i.e. bisimulation being a congruence, operations preserving semistochasticity, expressiveness. Moreover, we aim to develop rule formats for other behavioral equivalences, such as probabilistic weak bisimulation [5], and probabilistic testing equivalence [21. Finally, we aim to develop rule formats guaranteeing that security properties for probabilistic processes, such as those defined in [2, are respected by process algebra operations, on the same line followed in [17, 18, for classic process algebras.

\section{References}

1. L. Aceto, W. J. Fokkink, and C. Verhoef: Structural Operational Semantics. Handbook of Process Algebra, Elsevier, Amsterdam, 2001, 197-292.

2. A. Aldini, M. Bravetti, and R. Gorrieri: A Process-algebraic Approach for the Analysis of Probabilistic Non-interference. J. Comput. Secur. 12, 2004, 191-245.

3. J. C. M. Baeten, J. A. Bergstra, and S. A. Smolka: Axiomatizing Probabilistic Processes: ACP with Generative Probabilities. Inf. Comput. 121, 1995, 234-255.

4. J. C. M. Baeten and C. Verhoef: A Congruence Theorem for Structured Operational Semantics with Predicates. Proc. Concurrency Theory, LNCS 715, 1993.

5. C. Baier and H. Hermanns: Weak Bisimulation for Fully Probabilistic Processes. Proc. Computer Aided Verification, LNCS 1254, 1997, 119-130.

6. F. Bartels: GSOS for Probabilistic Transition Systems. Proc. Coalgebraic Methods in Computer Science, ENTCS 65, 2002.

7. B. Bloom, S. Istrail, and A. Meyer: Bisimulation Can't Be Traced. J. Assoc. Comput. Mach. 42, 1995, 232-268.

8. M. Bravetti and A. Aldini: Discrete Time Generative-reactive Probabilistic Processes with Different Advancing Speeds. Theor. Comput. Sci. 290, 2003, 355-406.

9. P. R. D'Argenio, H. Hermanns, and J. P. Katoen: On Generative Parallel Composition. Proc. Probabilistic Methods in Verification, ENTCS 22, 1999.

10. A. Giacalone, C.C. Jou, and S.A. Smolka: Algebraic Reasoning for Probabilistic Concurrent Systems. IFIP Work. Conf. on Progr., Concepts and Methods, 1990.

11. R. J. van Glabbeek, S. A. Smolka, and B. Steffen: Reactive, Generative and Stratified Models of Probabilistic Processes. Inf. Comput. 121, 1995, 59-80.

12. J. F. Groote and F. Vaandrager: Structured Operational Semantics and Bisimulation as a Congruence. Inf. Comput. 100, 1992, 202-260.

13. B. Jonsson, K. L. Larsen, and W. Yi: Probabilistic Extensions of Process Algebras. Handbook of Process Algebra, Elsevier, Amsterdam, 2001.

14. G. Plotkin: A Structural Approach to Operational Semantics. Technical report DAIMI FN-19, University of Aarhus, 1981.

15. G. Plotkin: A Structural Approach to Operational Semantics. J. Log. Algebr. Program. 60-61, 2004, 17-139.

16. R. de Simone: Higher-level Synchronizing Devices in Meije-SCCS. Theor. Comput. Sci. 37, 1985, 245-267. 
17. S. Tini: Rule Formats for Non-Interference. Proc. European Symp. on Programming, LNCS 2618, 2003, 129-143.

18. S. Tini: Rule Formats for Compositional non Interference Properties. J. Log. Algebr. Program. 60-61, 2004, 353-400.

19. I. Ulidowski and I. Phillips: Ordered SOS Process Languages for Branching and Eager Bisimulations. Inf. Comput. 178, 2002, 180-213.

20. C. Verhoef: A Congruence Theorem for Structural Operational Semantics with Predicates and Negative Premises. Nord. J. Comput. 2, 1995, 274-302.

21. S. H. Wu, S. A. Smolka, and E. W. Stark: Composition and Behaviors of Probabilistic I/O Automata. Theor. Comput. Sci. 176, 1997, 1-38. 